ARTICLE

\title{
Sub-thermionic, ultra-high-gain organic transistors and circuits
}

Zhongzhong Luo ${ }^{1,13}$, Boyu Peng ${ }^{2,3,13}$, Junpeng Zeng ${ }^{1,13}$, Zhihao Yu14,13, Ying Zhao5 , Jun Xie ${ }^{6}$, Rongfang Lan ${ }^{6}$,

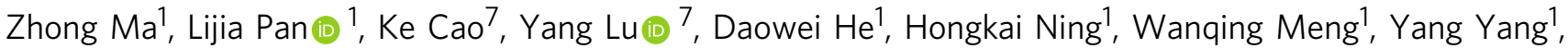
Xiaoqing Chen", Weisheng $\mathrm{Li}^{1}$, Jiawei Wang ${ }^{5}$, Danfeng Pan ${ }^{1,8}$, Xuecou Tu (1) ${ }^{1,8}$, Wenxing Huo ${ }^{9}$, Xian Huang ${ }^{9}$, Dongquan Shi ${ }^{10}$, Ling $\mathrm{Li}^{5}$, Ming Liu ${ }^{5}$, Yi Shi (i) ${ }^{1}$, Xue Feng ${ }^{11}$, Paddy K. L. Chan (1D) ${ }^{2,12 \bowtie} \&$ Xinran Wang (i) ${ }^{1 凶}$

The development of organic thin-film transistors (OTFTs) with low power consumption and high gain will advance many flexible electronics. Here, by combining solution-processed monolayer organic crystal, ferroelectric $\mathrm{HfZrO}_{x}$ gating and van der Waals fabrication, we realize flexible OTFTs that simultaneously deliver high transconductance and sub- $60 \mathrm{mV} / \mathrm{dec}$ switching, under one-volt operating voltage. The overall optimization of transconductance, subthreshold swing and output resistance leads to transistor intrinsic gain and amplifier voltage gain over $5.3 \times 10^{4}$ and $1.1 \times 10^{4}$, respectively, which outperform existing technologies using organics, oxides and low-dimensional nanomaterials. We further demonstrate batterypowered, integrated wearable electrocardiogram (ECG) and pulse sensors that can amplify human physiological signal by 900 times with high fidelity. The sensors are capable of detecting weak ECG waves (undetectable even by clinical equipment) and diagnosing arrhythmia and atrial fibrillation. Our sub-thermionic OTFT is promising for battery/wireless powered yet performance demanding applications such as electronic skins and radio-frequency identification tags, among many others.

\footnotetext{
${ }^{1}$ National Laboratory of Solid-State Microstructures, School of Electronic Science and Engineering and Collaborative Innovation Center of Advanced Microstructures, Nanjing University, Nanjing 210093, China. ${ }^{2}$ Department of Mechanical Engineering, The University of Hongkong, Pok Fu Lam Road, Hong Kong, China. ${ }^{3}$ MOE Key Laboratory of Macromolecular Synthesis and Functionalization, Department of Polymer Science and Engineering, Zhejiang University, Hangzhou 310027, China. ${ }^{4}$ College of Electronic and Optical Engineering, Nanjing University of Posts and Telecommunications, Nanjing 210023, China. ${ }^{5}$ Key Laboratory of Microelectronics Devices and Integrated Technology, Institute of Microelectronics, Chinese Academy of Sciences, Beijing 100029, China. ${ }^{6}$ Department of Cardiology, Nanjing Drum Tower Hospital, The Affiliated Hospital of Nanjing University Medical School, Nanjing 210093, China. ${ }^{7}$ Department of Mechanical Engineering, City University of Hong Kong, Kowloon, Hong Kong, China. ${ }^{8}$ Microfabrication and Integration Technology Center, Nanjing University, Nanjing 210093, China. ${ }^{9}$ Department of Biomedical Engineering, Tianjin University, Tianjin 300072, China. ${ }^{10}$ Department of Sports Medicine and Adult Reconstructive Surgery, Nanjing Drum Tower Hospital, The Affiliated Hospital of Nanjing University Medical School, Nanjing 210093, China. ${ }^{11}$ AML, Department of Engineering Mechanics, Center for Flexible Electronics Technology, Tsinghua University, Beijing 100084, China. ${ }^{12}$ Advanced Biomedical Instrumentation Centre, Hong Kong Science Park, Shatin, New Territories, Hong Kong, China. ${ }^{13}$ These authors contributed equally: Zhongzhong Luo, Boyu Peng,

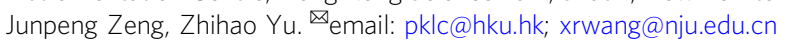


O rganic thin-film transistors (OTFTs) have been extensively pursued for printable and flexible electronic applications owing to their intrinsic flexibility and lowcost processing ${ }^{1-6}$. Many applications, such as Internet of Things (IoT) and wearable electronics, require low-voltage operation while providing high enough current to drive the circuits or high gain to amplify small signals. So far, however, there exist considerable challenges to make OTFT competitive over carbon nanotube films ${ }^{7}$, two-dimensional (2D) materials ${ }^{8}$ and oxides ${ }^{9}$. First, the mobility of organic semiconductors is generally lower than their inorganic counterparts. This leads to low transconductance $\left(g_{\mathrm{m}}\right)$ and intrinsic gain $\left(A_{\mathrm{i}}=g_{\mathrm{m}} \cdot r_{0}\right.$, where $r_{0}$ is output resistance). Second, the contact resistance, which partly arises the vertical access resistance from the finite film thickness and disorders introduced by conventional fabrication processes (such as lithography and metal deposition), is a major limiting factor towards high-frequency operation ${ }^{10}$. Third, the switching of OTFTs is often far from ideal, which results in large operating voltage $\left(V_{\mathrm{dd}}\right)^{11}$. Despite significant efforts, it remains a challenge to maintain subthreshold swing (SS) close to the Boltzmann thermionic limit $\left(\ln (10) \frac{k_{B} T}{q} \approx 60 \mathrm{mV} / \mathrm{dec}\right.$, where $q$ is elementary charge, $k_{\mathrm{B}}$ is Boltzmann's constant, and $T$ is temperature) over extended range and $V_{\mathrm{dd}}$ low enough for complete battery/wireless operation $^{12-14}$

Recently, negative capacitance (NC) effect from ferroelectric hafnium oxides provides a promising solution for low-power and complementary metal-oxide-semiconductor (CMOS) compatible electronics ${ }^{15-24}$. Experimentally, NC transistors have been implemented on silicon ${ }^{25-27}$, germanium ${ }^{28}$ and $2 \mathrm{D}$ materials ${ }^{29,30}$, showing evidence of sub- $60 \mathrm{mV} / \mathrm{dec}$ switching and enhanced $g_{\mathrm{m}}$. Moreover, a transition from negative to positive drain-induced barrier lowing (DIBL) was theoretically predicted and experimentally observed in NC transistors ${ }^{16,29}$. This would lead to infinite $r_{0}$ and intrinsic gain near the transition region, which is desirable for high-gain analog amplifiers. However, this unique advantage of NC transistors has not been fully exploited so far.

Here, we combine solution-processed OTFT with ferroelectric $\mathrm{HfZrO}_{\mathrm{x}}(\mathrm{HZO})$ gating to demonstrate flexible sub-thermionic organic transistors as well as ultra-high-gain amplifier circuits. Our technology has several unique features. First, the monolayer organic crystal film ensures excellent gate control while maintaining high channel mobility up to $10.4 \mathrm{~cm}^{2} \mathrm{~V}^{-1} \mathrm{~s}^{-1}$. Second, the double-well energy landscape of ferroelectric gating and associated NC effect break the thermionic limit in $S S(60 \mathrm{mV} / \mathrm{dec})$ and transconductance efficiency (38.7 S/A) and simultaneously enhances on-current and $g_{\mathrm{m}}$ by saving overdrive voltage loss $15,23,29,30$. In addition, the negative DIBL introduced by ferroelectric $\mathrm{HZO}^{16,29}$ produces large $r_{0} \sim 10^{10} \Omega$. Third, the uniform film morphology and van der Waals (vdW) integration of metal electrodes $^{31}$ ensure direct and damage-free contact with the channel, giving contact resistance below $60 \Omega \mathrm{cm}$. Together, these systematic optimizations not only significant improve the performance and power consumption of OTFTs, but also deliver the highest gain for any TFT technologies. Finally, our subthermionic OTFTs are low-temperature solution processed, scalable to large area and compatible with flexible substrates, which allow us to fabricate integrated wearable sensors health monitoring and diagnosis.

\section{Results}

Device fabrication and characterizations. For device fabrication, we first grew $\mathrm{HZO} / \mathrm{Al}_{2} \mathrm{O}_{3}$ gate dielectrics on highly doped silicon wafers by atomic layer deposition (ALD) and carried out solution shearing of p-type 2,9-didecyldinaphtho[2,3-b:2',3'-f] thieno[3,2b] thiophene $\left(\mathrm{C}_{10}\right.$-DNTT) as channel material ${ }^{32,33}$ (see "Methods" section for experimental details). The $\mathrm{Hf}: \mathrm{Zr}$ atomic ratio was close to $1: 1$ as revealed by X-ray photoelectron spectroscopy (Supplementary Fig. 1b). The HZO showed low surface roughness $(\sim 0.3 \mathrm{~nm})$ and ferroelectric properties as confirmed by atomic force microscopy (AFM), piezoelectric force microscopy, and polarization-electric field (P-E) loop measurements (Supplementary Fig. 1). Highly uniform monolayer organic films were obtained by optimizing the substrate temperature and blade speed in the shearing process ${ }^{34}$ (Supplementary Fig. 2). Figure 1a, f show optical images of monolayer $\mathrm{C}_{10}$-DNTT films on 2 -in. silicon wafer and polyimide, respectively. The film thickness was characterized by ellipsometry and AFM, both showing $\sim 4 \mathrm{~nm}$ (corresponding to monolayer) with excellent wafer-scale uniformity (Fig. 1b, c). The films were highly crystalline with herringbone lattice, consistent with $\mathrm{C}_{10}$-DNTT bulk crystals (Fig. 1d). Under optimized conditions, the domain size could reach several millimeters (Fig. 1e), which effectively reduced domain boundaries within device channel. The average fieldeffect mobility of monolayer OTFTs on $\mathrm{Al}_{2} \mathrm{O}_{3}$ dielectrics was $8.44 \pm 1.61 \mathrm{~cm}^{2} \mathrm{~V}^{-1} \mathrm{~s}^{-1}$ (Supplementary Fig. 3), further proving the high quality of $\mathrm{C}_{10}$-DNTT films. On ultra-thin polyimide substrate, the monolayer film was highly transparent (>97\%) and conformable to epidermis to allow the construction of skin-like wearable devices (Fig. 1g, Supplementary Figs. 2b and 4).

It is well-known that conventional OTFT fabrication processes can damage the organic films, especially for monolayer ${ }^{35}$. To this end, we developed solvent-free and low-energy vdW fabrication process as illustrated in Supplementary Fig. 5. This approach is readily scalable to wafer-scale with nearly $100 \%$ yield and micrometer resolution (Fig. 2a). Briefly, we first prepared patterned Au contact electrodes on a silicon wafer. The electrodes were mechanically released using PMMA and thermal release tape and gently laminated onto the pre-patterned channel areas. Such low-energy process preserved the integrity of monolayer $\mathrm{C}_{10}$-DNTT channel and contact interface as shown by the cross-sectional transmission electron microscopy (TEM) image (Fig. 2b). After removing the tape, electron-beam lithography (EBL) was performed to remove the PMMA on probing pads for electrical measurements. The intimate and damage-free contact to the monolayer channel indeed led to contact resistance of $59.4 \pm$ $5.3 \Omega \mathrm{cm}$ as measured by transfer length method (Supplementary Fig. 6), close to the record for OTFTs (Supplementary Table 1).

Electrical performance of sub-thermionic OTFTs and device modeling. Figure $2 \mathrm{c}$, d presents the room temperature transfer $\left(I_{\mathrm{ds}}-V_{\mathrm{gs}}\right)$ and output $\left(I_{\mathrm{ds}}-V_{\mathrm{ds}}\right)$ characteristics of a typical subthermionic monolayer OTFT under $V_{\mathrm{dd}}=1 \mathrm{~V}$. We observed several remarkable features, including extended subthreshold region with a steep slope, close-to-zero threshold voltage $\left(V_{\text {th }}\right)$, on/off ratio over $10^{8}$, excellent saturation behavior, and no hysteresis. The derived point $S S$ was below $60 \mathrm{mV} / \mathrm{dec}$ for more than two decades (Fig. 2e). The average $S S$ was 58.6 and $60.9 \mathrm{mV} / \mathrm{dec}$ for one and five decades of $I_{\mathrm{ds}}$, respectively. The device also delivered superior on-state performance. In particular, the normalized $g_{\mathrm{m}}$ reached high value of $0.41 \mu \mathrm{S} \mu \mathrm{m}^{-1}$ under one-volt operation (Supplementary Table 2), suggesting possible radiofrequency applications ${ }^{10}$. More importantly, the transconductance efficiency, $\frac{g_{m}}{I_{d s}}=41.6 \mathrm{~S} / \mathrm{A}$, exceeded the theoretical limit of $\frac{q}{k_{B} T}=38.7 \mathrm{~S} / \mathrm{A}^{4}$ imposed by Boltzmann distribution of carriers (Fig. 2e). The high transconductance efficiency was maintained over several orders of $I_{\mathrm{ds}}$, which was desirable in analog circuit design but difficult for $\mathrm{CMOS}^{36}$. To evaluate the device variations, we measured 50 OTFTs (the transfer characteristics and statistics are shown in Supplementary Fig. 7). Figure $2 \mathrm{f}$ presents the histogram of $S S$ (over one decade of $I_{\mathrm{ds}}$ rather than point $S S$ ), 
a

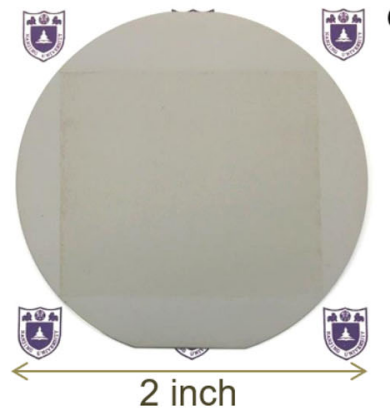

C

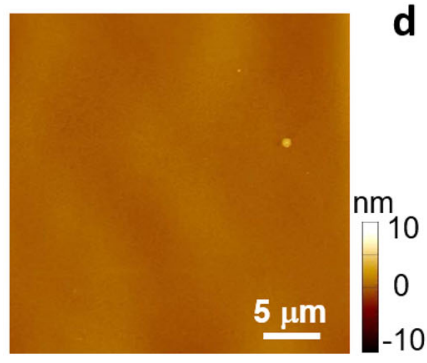

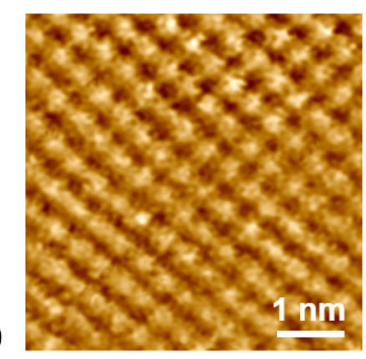

e

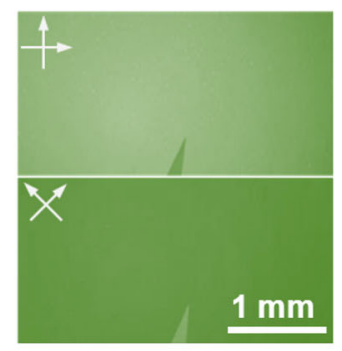

b

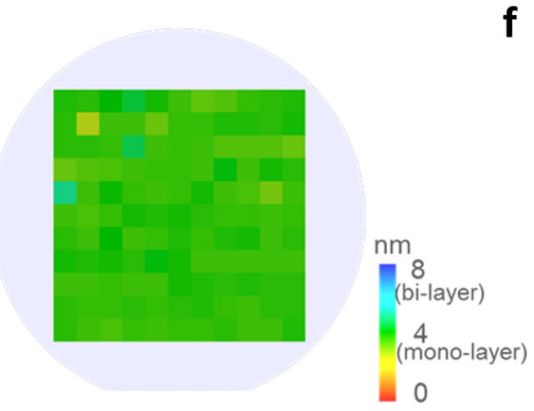

$\mathbf{f}$

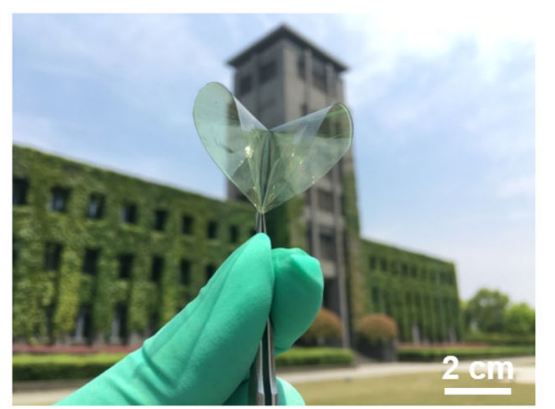

g

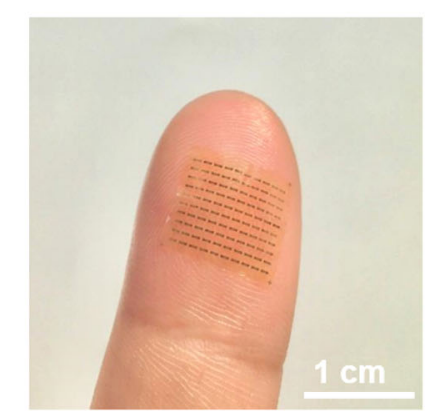

Fig. 1 Large-area monolayer $\mathbf{C}_{\mathbf{1 0}}$-DNTT films. a Photograph of uniform monolayer $C_{10}$-DNTT film on 2-in. Si wafer. $\mathbf{b}$ The thickness mapping of the sample in a using spectral ellipsometry. AFM (c), high-resolution AFM (d) and cross-polarized optical micrograph (e) of the monolayer $\mathrm{C}_{10}$-DNTT film. Photograph of monolayer $\mathrm{C}_{10}$-DNTT film on polyimide substrate (f) and flexible device array laminated on a fingertip ( $\mathbf{g}$ ).

showing Gaussian-like distribution with $2.5 \%$ variation as measured from the full-width at half-maximum. Sub- $60 \mathrm{mV} / \mathrm{dec}$ switching was observed in 68\% (34 out of 50) devices.

To demonstrate the advantage of sub-thermionic OTFTs, we fabricated monolayer OTFTs on $24 \mathrm{~nm} \mathrm{Al}_{2} \mathrm{O}_{3} / \mathrm{Si}$ substrate (without HZO). The minimum SS of these "normal" devices was $65-75 \mathrm{mV} / \mathrm{dec}$ and degraded quickly with $I_{\mathrm{ds}}$ (Supplementary Fig. 8a). Consequently, sub-thermionic OTFTs delivered more than one order of magnitude higher on-current and $g_{\mathrm{m}}$ under the same $V_{\mathrm{dd}}$ (Supplementary Fig. 8). These observations were consistent with a recent theoretical model based on realistic capacitance analysis ${ }^{23}$.

To gain further insight of the device physics, we performed quantitative modeling using an equivalent circuit model in Supplementary Fig. 9. To achieve simultaneous hysteresis-free and sub-thermionic switching, the ferroelectric $\mathrm{HZO}$ capacitance $\left(C_{\mathrm{FE}}\right)$ needs to fall between that of $\mathrm{Al}_{2} \mathrm{O}_{3} \quad\left(C_{\mathrm{AlOx}}\right)$ and $C_{\mathrm{MOS}} \approx\left(C_{\mathrm{AlO}_{\mathrm{x}}}^{-1}+\left(C_{\mathrm{j}, \mathrm{S}}+C_{\mathrm{j}, \mathrm{D}}\right)^{-1}\right)^{-1}$, where $C_{\mathrm{j}, \mathrm{S} / \mathrm{D}}$ are $\mathrm{S} / \mathrm{D}$ Schottky junction capacitances (Supplementary Fig. 9) ${ }^{23}$. The low dielectric constant and low channel doping of organic channel generate $C_{\mathrm{j}, \mathrm{S} / \mathrm{D}} \approx 6 \times 10^{-7} \mathrm{~F} / \mathrm{cm}^{2}$, which is about one order of magnitude smaller than $C_{\mathrm{AlOx}}\left(3.6 \times 10^{-6} \mathrm{~F} / \mathrm{cm}^{2}, 2 \mathrm{~nm}\right.$ $\mathrm{Al}_{2} \mathrm{O}_{3}$ ). This defines an extended design space for $C_{\mathrm{FE}}$. The thin $\mathrm{Al}_{2} \mathrm{O}_{3}$ also reduces the minimum thickness of $\mathrm{HZO}$ to achieve sub-60 mV/dec switching. Supplementary Figure 9d (solid blue line) plots $S S$ as a function of $C_{F E}^{-1}$ using our experimental device geometry. The $C_{\mathrm{FE}}$ of $22 \mathrm{~nm} \mathrm{HZO}$ (derived from fitting of P-E loop, Supplementary Fig. 1d) gives $S S=56.5 \mathrm{mV} / \mathrm{dec}$ (red star), in good agreement with experimental data. The correct capacitance matching of sub-thermionic OTFTs also makes the hysteresis of $I_{\mathrm{ds}}-V_{\mathrm{gs}}$ curve negligible ${ }^{16,23}$. Furthermore, we quantitatively calculated the transistor characteristics using a compact model incorporating time-dependent depolarization and multi-domain Landau-Khalatnikov theory ${ }^{37}$. Supplementary Figure 9e, f shows that the calculated transfer and output curves match experiments very well, which validates the above model analysis.

We further benchmark our sub-thermionic OTFTs with other TFT technologies. Figure $2 \mathrm{~g}$ compares the $g_{\mathrm{m}}$ and on/off ratio (data from ten devices) with several representative OTFT technologies, under the same $\left|V_{\mathrm{gs} \text { (on) }}-V_{\mathrm{gs}(\mathrm{off})}\right|=1 \mathrm{~V}$ (see Supplementary Table 2 for details). Our data points are located at the upper right corner of the plot, exhibiting significantly improved $g_{\mathrm{m}}$ while maintaining on/off ratio greater than $10^{8}$. Figure $2 \mathrm{~h}$ summarizes the intrinsic gain of various TFT technologies. Our monolayer sub-thermionic OTFTs exhibit the highest $A_{\mathrm{i}}$ of $5.3 \times 10^{4}$, which is more than one order of magnitude higher than oxides ${ }^{38}$ and Schottky barrier TFTs ${ }^{4,39}$, and more than two orders of magnitude higher than $2 \mathrm{D}$ materials ${ }^{40}$ and silicon. Other than $g_{\mathrm{m}}$, an important reason for the high $A_{\mathrm{i}}$ is large $r_{0} \sim 10^{10} \Omega$ due to near-zero DIBL in the subthreshold region (Supplementary Fig. 10). This can be understood as a consequence of the transition from negative to positive DIBL, leading to infinite $r_{0}$ at the transition (Supplementary Fig. 10). This is a unique property of NC transistors ${ }^{16,29}$ and is also captured by our modeling (Supplementary Fig. 9g). It is important to note that the overall improvement of transistor performance $\left(g_{\mathrm{m}}, r_{0}, A_{\mathrm{i}}, S S\right)$ is accomplished by systematic optimization, including high mobility material, monolayer channel, clean contact interface and ferroelectric dielectrics. To verify the importance of monolayer channel, we fabricated devices with thicker channel and observed inferior SS, oncurrent and $g_{\mathrm{m}}$ due to poor electrostatics and contact resistance ${ }^{35,41}$ (Supplementary Fig. 8).

The devices also had good temperature and operational stability. The sub-60 mV/dec switching was preserved after 2month storage at room temperature, with only slight shift of $V_{\text {th }}$ and decrease of on-current (Supplementary Fig. 11a). Variabletemperature measurements showed that the SS was below thermionic limit from 250 to $375 \mathrm{~K}$ (Supplementary Fig. 11e, f), owing to the high Curie temperature of the $\mathrm{HZO}^{19}$. In addition, 
a
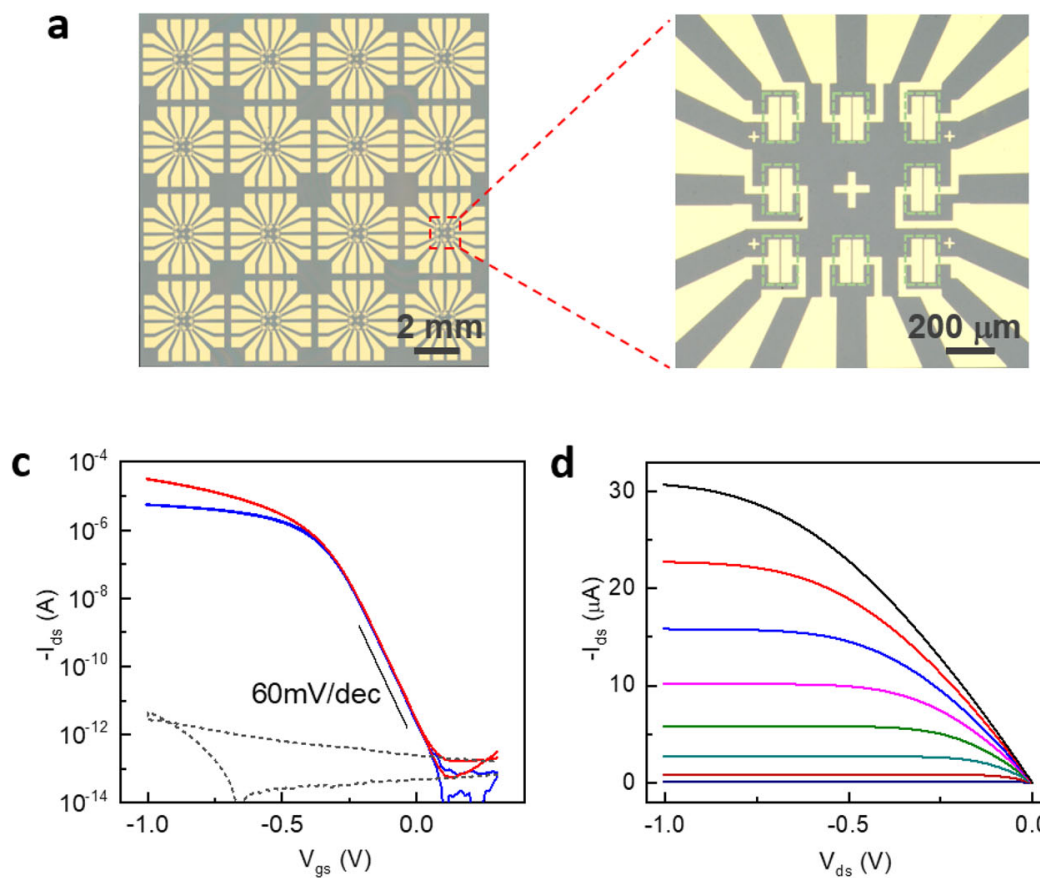

f

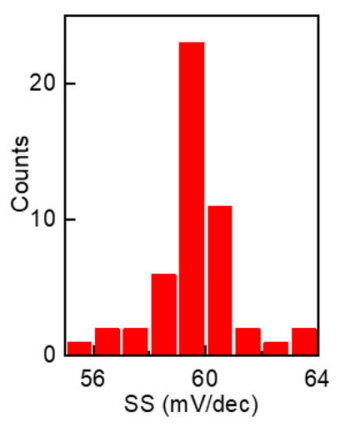

d

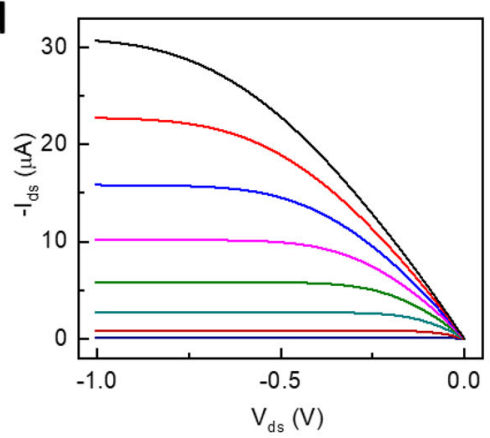

g

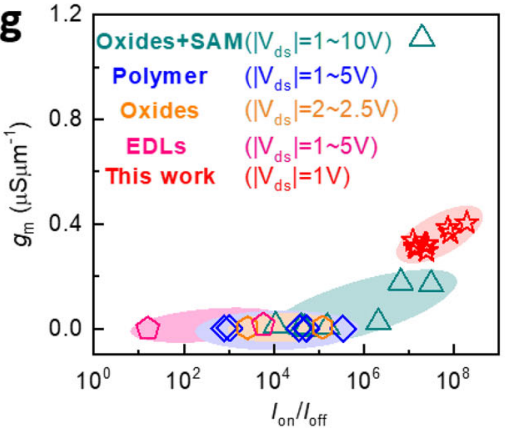

b

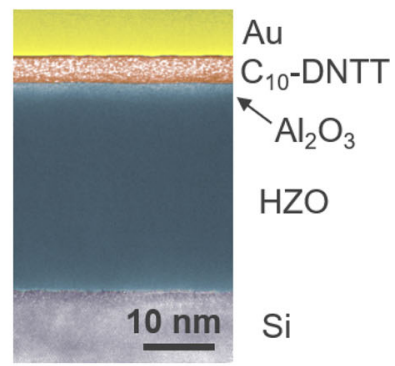

e
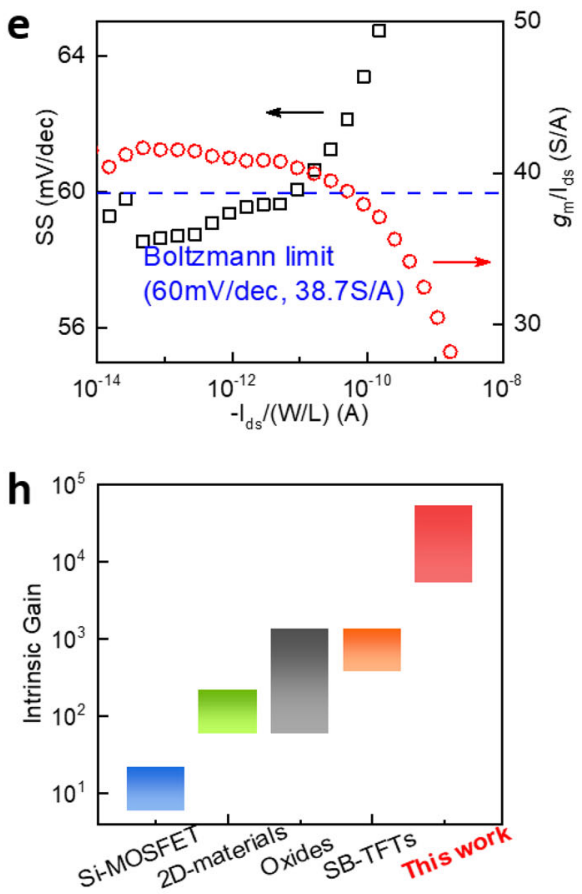

Fig. 2 Transistor characteristics of sub-thermionic monolayer OTFTs. a Photographs of device arrays fabricated on silicon substrate. The length to width ratio $(L / W)$ of device channel is $5 \mu \mathrm{m} / 180 \mu \mathrm{m}$. b False-color cross-section TEM image of a device contact region, clearly showing the Au/ $\mathrm{C}_{10}$-DNTT/ $\mathrm{Al}_{2} \mathrm{O}_{3} / \mathrm{HZO} / \mathrm{Si}$ stack. c Double-sweep $I_{\mathrm{ds}}-V_{\mathrm{gs}}$ curves of the sub-thermionic OTFT at $V_{\mathrm{ds}}=-0.1 \mathrm{~V}$ (blue) and $-1 \mathrm{~V}$ (red). No hysteresis is observed. The black-dashed line is the gate leakage current. $\mathbf{d} I_{\mathrm{ds}}-V_{\mathrm{ds}}$ curves of the sub-thermionic OTFT. From bottom to top, $V_{\mathrm{gs}}$ is from -0.3 to $-1 \mathrm{~V}$. e $\mathrm{SS}$ (black) and transconductance efficiency (red) as a function of $I_{\mathrm{ds}}$ for the device in c. The Boltzmann limit reference corresponds to $300 \mathrm{~K}$ under which our measurements were taken. $\mathbf{f}$ Statistical distributions of one-decade SS for 50 devices. $\mathbf{g}$ Comparison of $I_{\text {on }} / I_{\text {off }}$ and normalized transconductance at $\mid V_{g s}$ (on) $-V_{\mathrm{gs}(\mathrm{off})} \mid=1 \mathrm{~V}$ among OTFT technologies using different dielectrics. The extracted data are listed in Supplementary Table 2. $\mathbf{h}$ Comparison of intrinsic gain among different TFT technologies.

continuous bias stress measurements under $V_{\mathrm{ds}}=V_{\mathrm{gs}}=-1.5 \mathrm{~V}$ also showed little degradation of device performance after $10^{4} \mathrm{~s}$ (Supplementary Fig. 11b, c). Further, we conducted cycle stability test for the transistors, showing excellent cycle stability under repeated gate voltage pulses with $1 \mathrm{~Hz}$ frequency (Supplementary Fig. 11d). The stability can be further improved by proper passivation. In terms of frequency limit, previous studies have shown that $\mathrm{HZO}$ can properly function up to $\mathrm{MHz}^{42}$, which is adequate for OTFT applications.

Ultra-high-gain amplifier circuits. By designing proper electrode patterns, we could build functional circuits, such as inverter and logic gates using local backgate devices (Fig. 3, Supplementary Fig. 12, see "Methods" section for detailed fabrication processes). There is practically no limit in the circuit complexity by vdW integration as long as the transistors are coplanar. For very large-scale integration that requires multiple layers of interconnects, the upper layers can be fabricated by conventional lithography or printing as they are separated from the channel. Figure 3a shows the circuit and optical image of an enhancement-depletion mode inverter using two sub-thermionic OTFTs with different dimensions (the transfer characteristics are presented in Supplementary Fig. 13a). The inverter exhibited full swing output near zero input voltage with peak power of $\sim 50 \mathrm{nW}$ (Fig. 3b and Supplementary Fig. 13b). Remarkably, we obtained giant voltage gain $\left(A_{\mathrm{v}}\right)$ of $4.1 \times 10^{3}\left(1.1 \times 10^{4}\right)$ under $V_{\mathrm{dd}}=-1 \mathrm{~V}$ $(-3 \mathrm{~V})$ (Fig. 3c). On the other hand, control amplifiers fabricated on $\mathrm{Al}_{2} \mathrm{O}_{3} / \mathrm{Si}$ substrate showed 40 times lower $A_{\mathrm{v}}$ (Supplementary Fig. 13c, d). Therefore, we conclude that the high gain is enabled by steep switching in the transistor characteristics, which are unique properties of sub-thermionic OTFTs. Figure $3 \mathrm{~d}$ benchmarks the $A_{\mathrm{v}}$ of similar amplifier structures based on organics, metal oxides, 2D materials and carbon nanotubes (see Supplementary Table 3 for details). The gain of our devices again outperforms these existing technologies by orders of magnitude while maintaining low $V_{\mathrm{dd}}$, which hold promise for wearable electronics and IoT applications. 
a

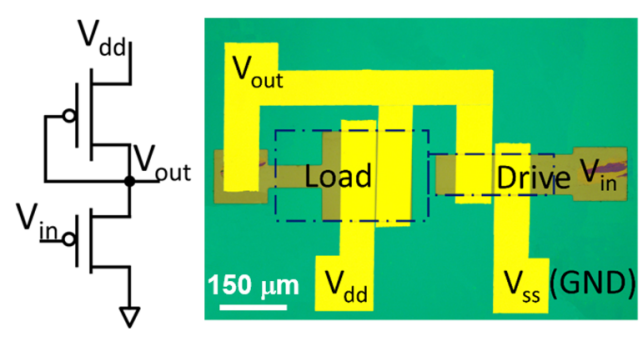

C

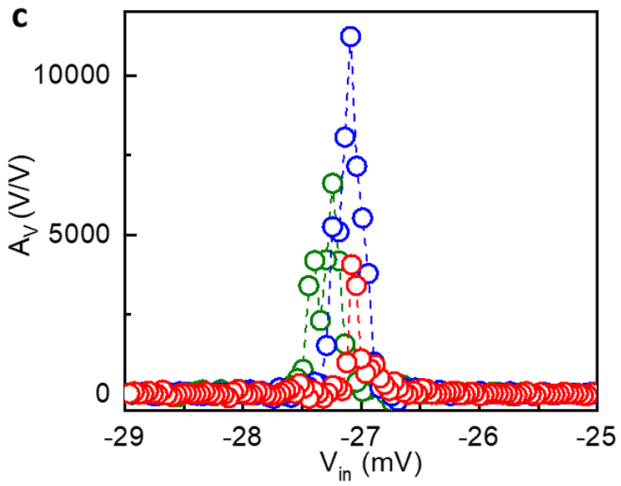

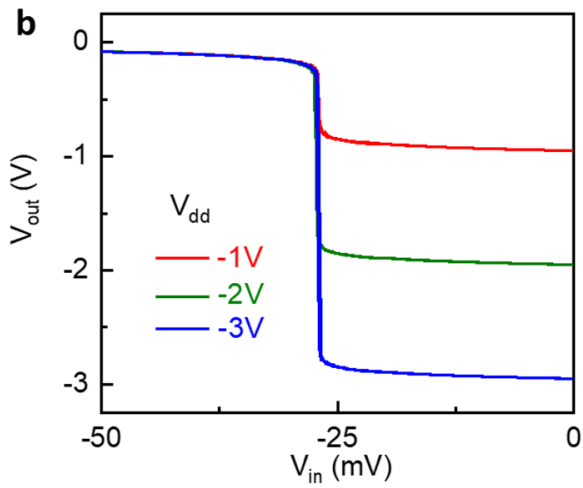

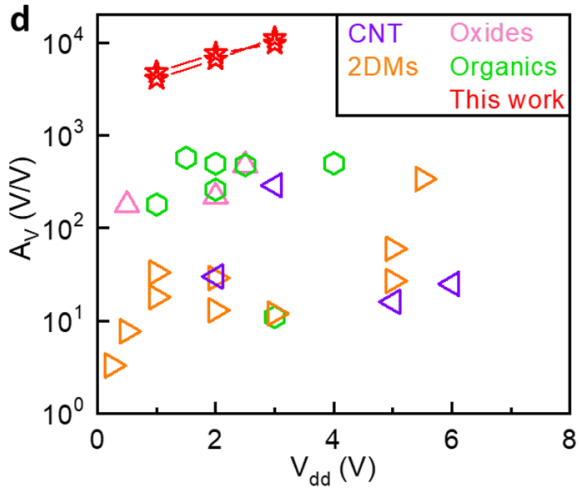

Fig. 3 Integrated amplifier based on sub-thermionic monolayer OTFTs. a Equivalent circuit diagram (left) and optical microscope image (right) of an enhancement-depletion mode amplifier. The dotted boxes indicate organic film areas for load and drive transistors. $\mathbf{b} \vee$ oltage transfer characteristics under $V_{\mathrm{dd}}=-1,-2$ and $-3 \mathrm{~V}$, respectively. $\mathbf{c} A_{\mathrm{v}}$ as a function of input voltage derived from $\mathbf{b}$. $\mathbf{d}$ Comparison of $A_{\mathrm{v}}$ among similar amplifier circuits using different materials and processes. The extracted data are listed in Supplementary Table 3.

Flexible sub-thermionic OTFTs and circuits. For wearable applications, it is important to examine the device performance on flexible substrates. To this end, polyimide was used because it was compatible with the processing temperature of HZO. Spincoated polyimide $(\sim 10 \mu \mathrm{m}$ thick) on sacrificial $\mathrm{Si}$ substrate ensured low surface roughness for solution shearing and mechanical reliability under extreme folding or crumpling. Figure 4a illustrates the structure and photograph of device arrays with local backgates. The transfer characteristics of 100 devices exhibit small variations in $V_{\text {th }}$ and $S S$ (Fig. $4 \mathrm{~b}, \mathrm{c}$ ). Importantly, the steep slope and high gain were preserved on polyimide substrate, with $75 \%$ (75 out of 100) devices exhibiting sub- $60 \mathrm{mV} / \mathrm{dec}$ switching and lowest $S S=56.5 \mathrm{mV} / \mathrm{dec}$ (Fig. 4c). Figure $4 \mathrm{~d}$ presents the transfer curves of a flexible inverter with $A_{\mathrm{v}}=9.7 \times 10^{3}$, which was comparable to that on Si substrate. Furthermore, the devices were robust under mechanical bending. Both $g_{\mathrm{m}}$ and SS showed little degradation during $10^{4}$ repeated bending tests to $10 \mathrm{~mm}$ radius of curvature (Fig. $4 \mathrm{e}$ ).

Integrated wearable ECG and pulse sensors. Next, we demonstrated the applications of sub-thermionic OTFT technology in wearable electrocardiogram (ECG) and pulse sensors. Human body constantly produces vital physiological signals, such as biopotentials and metabolites, in the range of microvolt to millivolt ${ }^{43-46}$. Therefore, on-site signal amplification using low-voltage and high-gain circuitry is essential for integrated wearable sensors but very challenging. We integrated a flexible enhancement-depletion mode amplifier on a custom-designed flexible circuit board, which could be easily laminated on the front chest (Fig. 5a, b). All the amplifiers were operated under $V_{\mathrm{dd}}=-1 \mathrm{~V}$ with the peak $A_{\mathrm{v}}$ greater than 1000 . The $V_{\mathrm{dd}}$ was supplied either by an external voltage source or a $1.5 \mathrm{~V}$ coin battery (Fig. $5 \mathrm{c}$, a voltage divider was used to reduce $V_{\mathrm{dd}}$ ), showing similar performance (Supplementary Fig. 14). Figure 5d plots the amplified ECG signal of a healthy male subject, clearly showing all the expected waveforms (P, Q, R, S, T and U waves). The signal amplitude was $\sim 350 \mathrm{mV}$, corresponding to an amplification factor of 324. The raw ECG signal could be obtained by deconvoluting the amplifier transfer curve (the blue solid line in Fig. 5e, see Supplementary Fig. 15 for details), which agreed well with the data recorded by a clinical grade commercial equipment (Prince $180 \mathrm{~B}$ by Heal Force, the gray-dashed line in Fig. 5e). Figure $5 \mathrm{~g}, \mathrm{~h}$ demonstrates continuous monitoring of ECG over several minutes. The signal fidelity, waveform and amplification factor remained stable during the test, which was essential for long-term health monitoring. To further prove the signal fidelity, we simultaneously measured the ECG signal by our sensor and commercial equipment (Supplementary Movie 1). Figure $5 f$ compares the power spectral density. We can see that the amplified ECG shares the same waveform with the commercial equipment, with the most prominent peak at $1.29 \mathrm{~Hz}$ corresponding to the heart rate of the subject. Importantly, the amplitude of power density was enhanced by $\sim 10^{5}$ times, consistent with the amplification factor of $\sim 300$. Battery-powered ECG also showed similar power spectral density.

With excellent sensitivity and signal fidelity, our ECG sensors are capable of facilitating cardiopathy diagnoses. To this end, two hospital volunteers were tested. On subject A (female, mid-20s) who was diagnosed with arrhythmia, our sensor clearly captured the irregular heartbeat with a sudden change from 83 to 43 beats per minute (bpm) (Fig. 5i). On subject B (male, $80 \mathrm{~s}$ ) who was diagnosed with atrial fibrillation (AF), our sensor was capable of detecting $\mathrm{f}$ wave with a frequency of $357 \mathrm{bpm}$, which was a direct 
a

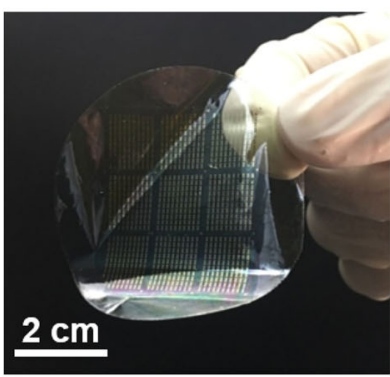

C

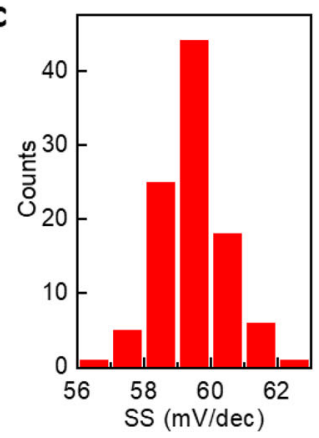

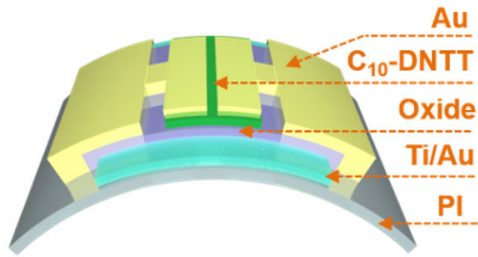
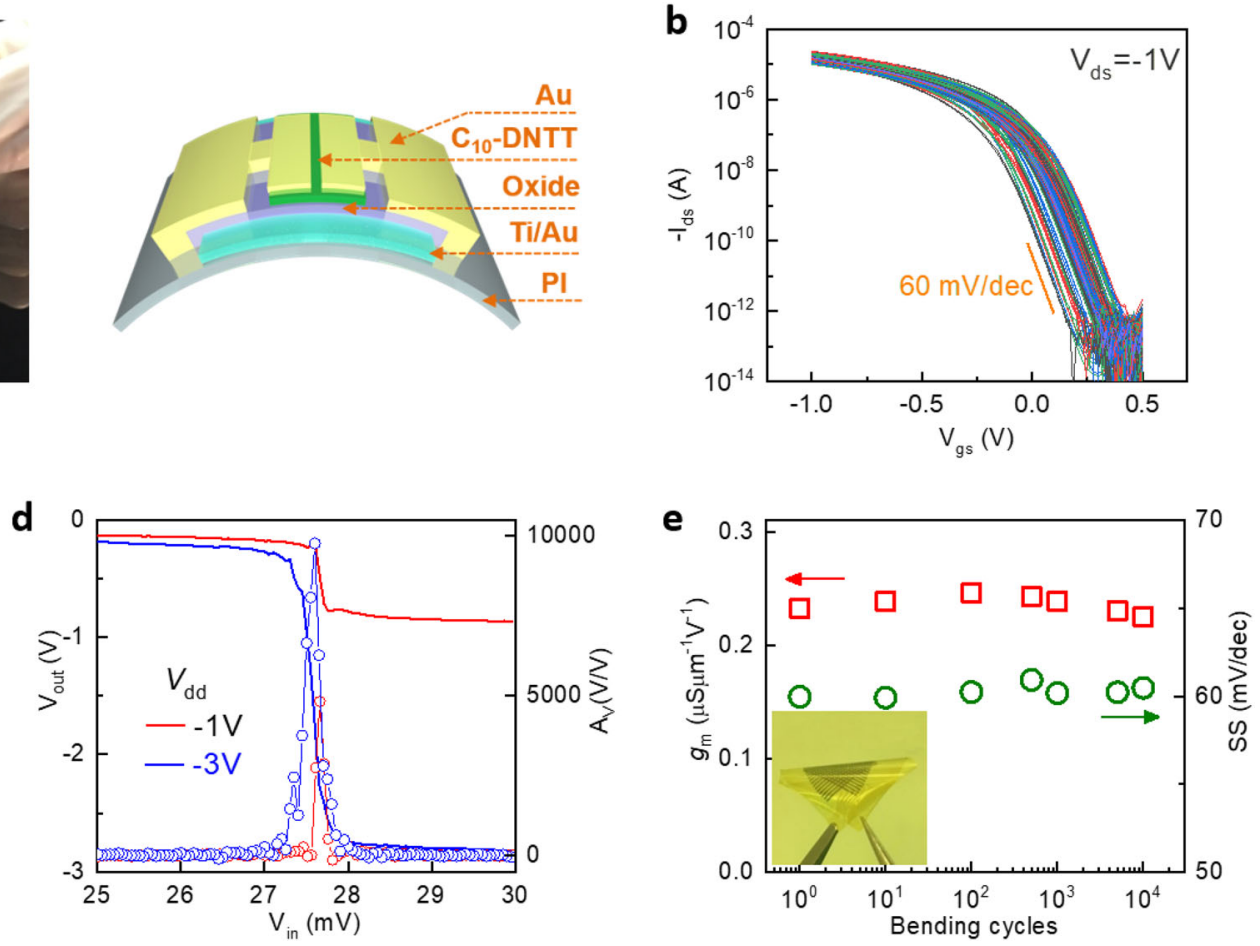

Fig. 4 Sub-thermionic OTFTs on flexible substrates. a Photograph of OTFT arrays fabricated on 2-in. polyimide substrate (left) and schematic view of the device structure (right). $\mathbf{b} I_{\mathrm{ds}}-V_{\mathrm{gs}}$ curves of 100 devices under $V_{\mathrm{ds}}=-1 \mathrm{~V}$. The $L / W$ of device channel is $5 \mu \mathrm{m} / 180 \mu \mathrm{m}$. c Statistical distributions of $S S$ for the devices in $\mathbf{b}$. $\mathbf{d}$ Voltage transfer characteristics (solid lines) and $A_{v}$ (symbols) of an enhancement-depletion mode amplifier on flexible substrate, under $V_{\mathrm{dd}}=-1$ and $-3 \mathrm{~V}$. e Bending test of a device on polyimide substrate. Both $g_{\mathrm{m}}$ and SS remain stable during $10^{4}$ bending cycles. Inset shows a photograph of flexible device arrays under bending.

signature of AF (Fig. 5j, lower panel). In contrast, the clinical ECG of same subject did not resolve $f$ wave due to its weak amplitude (Fig. 5j, upper panel, taken by NIHON KOHDEN ECG-1350P). Currently, AF is the most common arrhythmia globally associated with the increased risk of stroke and heart failure. The ability to directly identify $\mathrm{f}$ wave will significantly increase the sensitivity and specificity of clinical diagnosis. Without any post-processing, the signal-to-noise ratio (SNR) of our ECG signal reached $42 \mathrm{~dB}$, exceeding previously reported results 47,48 . The improved sensitivity and the ability to detect extremely weak signals are remarkable considering the simple circuity of our sensors.

As another example, we connected the amplifier to a flexible carbon nanotube sensor to measure wrist pulse signals (Fig. 5k). The pulse pressure-induced resistance change in the carbon nanotube film, resulting in a slight shift of amplifier input voltage. Figure 51 shows the measured pulse waveforms of a human subject with $\sim 120 \mathrm{mV}$ peak-to-valley amplitude, corresponding to amplification factor of 900 . The details of the pulse waveforms including a systolic peak, a dicrotic notch, a reflected systolic peak and an end-diastolic notch were clearly resolved. The higher amplification compared to ECG was due to the smaller input signal amplitude ( $\sim 0.13 \mathrm{mV}$ vs. $\sim 1 \mathrm{mV}$ for ECG), which allowed the amplifier to work at the peak gain point. Compared to literatures, our ECG and pulse sensors show advantages in terms of $V_{\mathrm{dd}}$, gain and SNR (Supplementary Table 4).

\section{Discussion}

We demonstrate the first sub-thermionic flexible OTFT technology by combining solution-processed monolayer organic channel, ferroelectric gate dielectrics and $\mathrm{vdW}$ device integration. The devices operate under one-volt and exhibit sub- $60 \mathrm{mV} / \mathrm{dec}$ switching and record-high intrinsic gain. We further build amplifier circuits with ultra-high voltage gain of $1.1 \times 10^{4}$, which outperform existing TFT technologies by more than one order of magnitude. The flexible amplifiers are capable of amplifying human physiological signals by several hundred times with high fidelity, and detecting extremely weak ECG signals to facilitate clinical diagnosis. By developing more robust amplification schemes (such as differential amplification) ${ }^{48}$ and wireless data transmission $^{49}$, we can envision all-organic, battery-powered patches for high-precision health monitoring and real-time cloud-based diagnosis.

\section{Methods}

Preparation of $\mathbf{H Z O} / \mathbf{A l}_{\mathbf{2}} \mathbf{O}_{\mathbf{3}}$ dielectrics. The $\mathrm{HZO}$ film was deposited by ALD on $\mathrm{p}++$ silicon (100) substrate at $200^{\circ} \mathrm{C}$ and base pressure of $\sim 1 \mathrm{~Pa}$ using Tetrakis (dimethylamido) hafnium as $\mathrm{Hf}$ source, Tetrakis(dimethylamido) zirconium as $\mathrm{Zr}$ source and $\mathrm{H}_{2} \mathrm{O}$ as oxidant. We used $20 \mathrm{sccm} \mathrm{N}$ as carrier gas. The $\mathrm{HfO}_{\mathrm{x}}$ and $\mathrm{ZrO}_{\mathrm{x}}$ were deposited sequentially with 1:1 ratio. Then $2 \mathrm{~nm} \mathrm{AlO}_{\mathrm{x}}$ capping layer was deposited by ALD in situ for capacitance matching, using trimethylaluminium and $\mathrm{H}_{2} \mathrm{O}$ as sources. The pulse/purge time for $\mathrm{Hf}, \mathrm{Zr}$ and $\mathrm{H}_{2} \mathrm{O}$ precursors were $250 \mathrm{~ms} /$ $30 \mathrm{~s}, 20 \mathrm{~ms} / 30 \mathrm{~s}$ and $30 \mathrm{~ms} / 30 \mathrm{~s}$, respectively. The substrate was undergone $350^{\circ} \mathrm{C}$ rapid thermal annealing for $1 \mathrm{~min}$ in $\mathrm{N}_{2}$ to crystallize the HZO.

We fabricated the $\mathrm{HZO} / \mathrm{Al}_{2} \mathrm{O}_{3}$ dielectrics on polyimide substrate using the similar method mentioned above. To prepare the polyimide substrate, we spincoated polyimide solution (AA-49, KANEKA) on $\mathrm{SiO}_{2} / \mathrm{Si}$ substrate, and then baked at $350^{\circ} \mathrm{C}$ for $1 \mathrm{~h}$ on a hotplate.

Solution shearing of monolayer $\mathbf{C}_{\mathbf{1 0}}$-DNTT films. The $\mathrm{C}_{10}$-DNTT powder was dissolved in 1,2,3,4-tetrahydronaphthalene (tetralin) at $85^{\circ} \mathrm{C}$ with a concentration of $0.2 \mathrm{mg} / \mathrm{mL}$. The growth of monolayer $\mathrm{C}_{10}$-DNTT was carried out in a homebuilt solution shearing setup with heaters on both the blade and substrate (Supplementary Fig. 2). The temperature of the blade and substrate was set to $65^{\circ} \mathrm{C}$. Overall, $150 \mu \mathrm{L}$ of $\mathrm{C}_{10}$-DNTT solution was injected for a 2 in. size substrate and a constant shear velocity of $3 \mu \mathrm{m} / \mathrm{s}$ was established by using a linear translation stage during the shearing processes. 
a

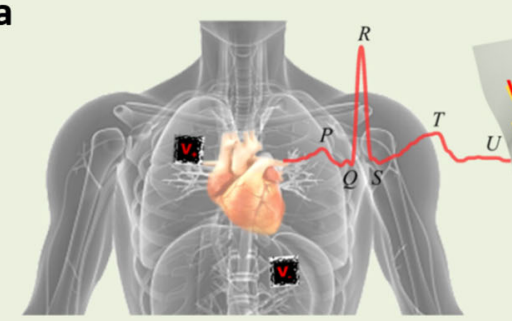

b

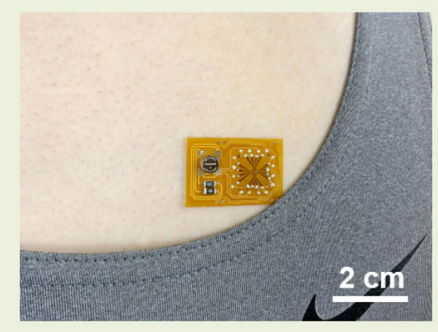

d

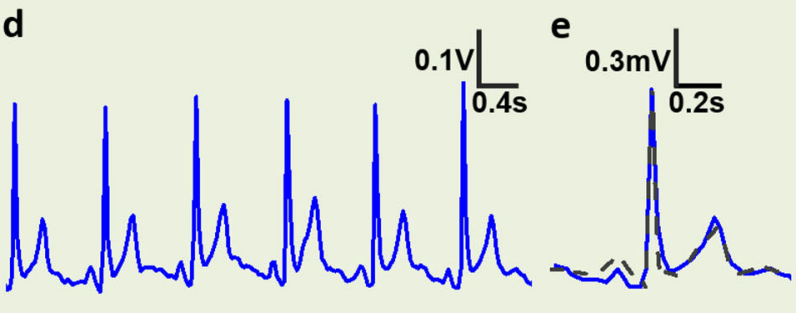

C

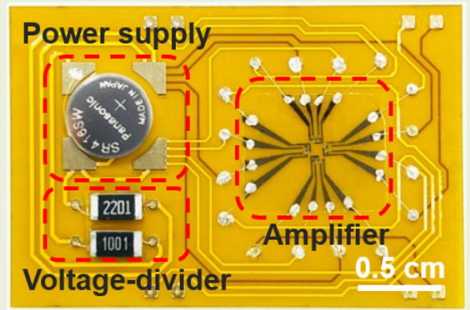

f

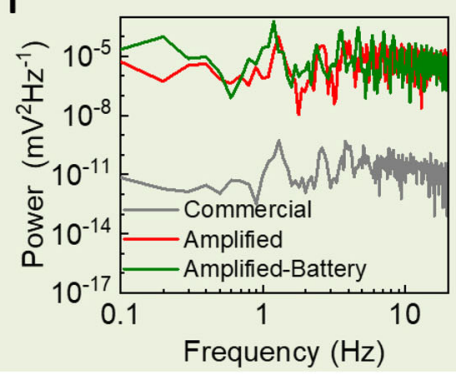

k

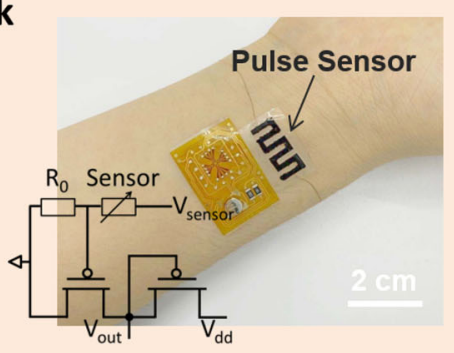

I

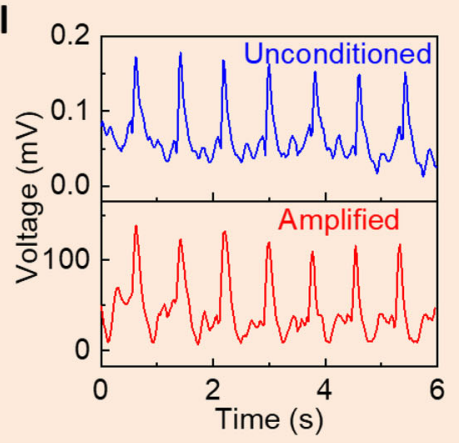

Fig. 5 Low-voltage- and high-gain-integrated wearable sensors. a Cartoon illustration of the use of amplifier circuit to acquire human ECG signals. b Photograph of a flexible amplifier module attached to a human chest. c Photograph of the amplifier module, with a coin battery, a voltage divider and amplifier circuit integrated on a flexible circuit board. d Amplified ECG signal of a human subject. e Deconvoluted ECG signal using the amplifier's transfer curve (blue solid line) and the ECG signal taken by commercial equipment on the same human subject (black-dashed line). $\mathbf{f}$ The power spectral density of ECG signals taken by commercial equipment (gray), our amplifier with external voltage source (red) and battery (green) as power supply. $\mathbf{g}$ Long-term ECG monitoring using our sensor. $\mathbf{h}$ The ECG signal of the 1st (upper panel) and 245th (lower panel) cycle in $\mathbf{g}$. $\mathbf{i}$ Amplified ECG signal of a human subject with known arrhythmia history (bottom panel) and the corresponding heart rate (upper panel). $\mathbf{j} E C G$ signal of another human subject with AF, recorded by our sensor (bottom panel) and a clinical equipment (upper panel). $\mathbf{k}$ Photograph of an integrated pulse sensor using flexible carbon nanotube films and our amplifier circuit. Inset shows the circuit diagram. I Unconditioned and amplified pulse signal taken on the same human subject.

Characterizations of $\mathbf{C}_{\mathbf{1 0}}$-DNTT film and OTFTs. We used ellipsometry mapping, high-resolution AFM and cross-polarized optical microscopy to characterize the monolayer $\mathrm{C}_{10}$-DNTT film. The ellipsometry mapping was performed on a

Woollam RC2-XI mapping ellipsometer in the wavelength range of $210-2500 \mathrm{~nm}$. For high-resolution AFM, the experiments were conducted on Asylum Cypher under ambient conditions. The cross-polarized optical reflection micrographs were performed by the ScanPro spectro-microscope under the white light. We used cross-sectional TEM to characterize the interface and thickness of sub-thermionic OTFTs. The TEM specimens were prepared using a FEI Scois Dualbeam focused ion beam. After that, the specimens were characterized using a high-resolution JEOL JEM-2100F TEM with acceleration voltage of $200 \mathrm{kV}$ equipped with energydispersive spectroscopy detector.

$\mathbf{v d W}$ integration of contacts. First, we prepare patterned gold electrodes with $50 \mathrm{~nm}$ thickness on a silicon substrate using EBL and electron-beam evaporation (EBE). Next, a PMMA layer was spin-coated, followed by the lamination of thermal released tape (TRT). The TRT/PMMA/Au electrode stack was carefully released 
from silicon substrate and was align transferred to patterned monolayer $\mathrm{C}_{10}$-DNTT film. Then the TRT was separated at $90^{\circ} \mathrm{C}$. Finally, the PMMA layer was patterned by EBL to remove the PMMA on probing pads for electrical measurements.

Fabrication of amplifiers with local backgate. First, we fabricated $10 \mathrm{~nm} \mathrm{Ti} / 20$ $\mathrm{nm}$ Au backgate electrodes on $\mathrm{SiO}_{2} / \mathrm{Si}$ substrate using EBL and EBE. Then, $\mathrm{HZO} /$ $\mathrm{Al}_{2} \mathrm{O}_{3}$ was deposited using ALD. EBL and inductive coupled plasma reactive ion etching $\left(\mathrm{BCl}_{3}\right.$ and $\left.\mathrm{Cl}_{2}\right)$ were adopted to etch $\mathrm{HZO} / \mathrm{Al}_{2} \mathrm{O}_{3}$ and open via holes to backgate electrodes. Then, monolayer $\mathrm{C}_{10}$-DNTT films were grown by the solution shearing process. Finally, the source/drain electrodes $(50 \mathrm{~nm} \mathrm{Au})$ were align transferred onto the $\mathrm{C}_{10}$-DNTT films. For the device on the polyimide substrate, the processes are similar.

Electrical measurements. Electrical measurements were carried out by an Agilent B1500 semiconductor parameter analyzer in a probe station under ambient conditions. And the temperature during the OTFT's electrical measurement was set at $300 \mathrm{~K}$.

Electrocardiogram and pulse detection. The flexible amplifier connected with two conventional gel electrodes to obtain the ECG signal. One was placed on the skin above the lower left part of heart and connected to a bias voltage to set the signal at the amplifier optimal work point. And another was placed on the upper right part of the heart as the input signal of the amplifier. We use Agilent B1500 to collect ECG signal and the sampling frequency was $40 \mathrm{~Hz}$. Fast Fourier transforms were applied to identify the power density of the recorded ECG signal. The SNR was calculated by the maximum peak value of the signal divided by the root mean square of the noise area. Inset of Fig. 5k shows the circuit diagram for pulse detection. The resistance of the flexible carbon nanotube sensor is about $1 \mathrm{k} \Omega$. By connecting it with a $1 \mathrm{k} \Omega$ divider resistor, the resistance change of the sensor was converted into a voltage signal, then as the input of the amplifier. Agilent B1500 was used to collect the pulse signal.

The authors affirm that human research participants provided informed consent for publication of the video in Supplementary Movie 1. All human subjects involved in the ECG and pulse tests provided informed consent, and the study protocol was approved by local ethics committee on human research of Nanjing Drum Tower Hospital (2019-190-01)

\section{Data availability}

All data that support the findings of this study are available from the corresponding author upon reasonable request.

Received: 13 October 2020; Accepted: 23 February 2021; Published online: 26 March 2021

\section{References}

1. Sirringhaus, H. 25th anniversary article: organic field-effect transistors: the path beyond amorphous silicon. Adv. Mater. 26, 1319-1335 (2014)

2. Kaltenbrunner, M. et al. An ultra-lightweight design for imperceptible plastic electronics. Nature 499, 458-463 (2013).

3. Wang, S. et al. Skin electronics from scalable fabrication of an intrinsically stretchable transistor array. Nature 555, 83 (2018).

4. Jiang, C. et al. Printed subthreshold organic transistors operating at high gain and ultralow power. Science 363, 719 (2019).

5. Klauk, H., Zschieschang, U., Pflaum, J. \& Halik, M. Ultralow-power organic complementary circuits. Nature 445, 745 (2007).

6. Wang, C., Dong, H., Jiang, L. \& Hu, W. Organic semiconductor crystals. Chem. Soc. Rev. 47, 422-500 (2018).

7. Tang, J. et al. Flexible CMOS integrated circuits based on carbon nanotubes with sub-10 ns stage delays. Nat. Electron. 1, 191-196 (2018).

8. Akinwande, D., Petrone, N. \& Hone, J. Two-dimensional flexible nanoelectronics. Nat. Commun. 5, 5678 (2014).

9. Myny, K. The development of flexible integrated circuits based on thin-film transistors. Nat. Electron. 1, 30-39 (2018).

10. Klauk, H. Will we see gigahertz organic transistors? Adv. Electron. Mater. 4, 1700474 (2018).

11. Liu, A., Zhu, H., Sun, H., Xu, Y. \& Noh, Y.-Y. Solution processed metal oxide high- $\kappa$ dielectrics for emerging transistors and circuits. Adv. Mater. 30, 1706364 (2018).

12. Borchert, J. W. et al. Flexible low-voltage high-frequency organic thin-film transistors. Sci. Adv. 6, eaaz5156 (2020).

13. Ji, D. et al. Copolymer dielectrics with balanced chain-packing density and surface polarity for high-performance flexible organic electronics. Nat. Commun. 9, 2339 (2018)
14. Yokota, T. et al. Flexible low-voltage organic transistors with high thermal stability at $250^{\circ} \mathrm{C}$. Adv. Mater. 25, 3639-3644 (2013).

15. Salahuddin, S. \& Datta, S. Use of negative capacitance to provide voltage amplification for low power nanoscale devices. Nano Lett. 8, 405-410 (2008).

16. Pahwa, G. et al. Analysis and compact modeling of negative capacitance transistor with high on-current and negative output differential resistancepart II: model validation. IEEE Trans. Electron Devices 63, 4986-4992 (2016)

17. Khan, A. I. et al. Negative capacitance in a ferroelectric capacitor. Nat. Mater 14, 182-186 (2015)

18. Böscke, T. S., Müller, J., Bräuhaus, D., Schröder, U. \& Böttger, U. Ferroelectricity in hafnium oxide thin films. Appl. Phys. Lett. 99, 102903 (2011).

19. Müller, J. et al. Ferroelectricity in simple binary $\mathrm{ZrO}_{2}$ and $\mathrm{HfO}_{2}$. Nano Lett. 12, 4318-4323 (2012).

20. Î́niguez, J., Zubko, P., Luk'yanchuk, I. \& Cano, A. Ferroelectric negative capacitance. Nat. Rev. Mater. 4, 243-256 (2019).

21. Alam, M. A., Si, M. \& Ye, P. D. A critical review of recent progress on negative capacitance field-effect transistors. Appl. Phys. Lett. 114, 090401 (2019).

22. Hoffmann, M. et al. Unveiling the double-well energy landscape in a ferroelectric layer. Nature 565, 464-467 (2019).

23. Cao, W. \& Banerjee, K. Is negative capacitance FET a steep-slope logic switch? Nat. Commun. 11, 196 (2020).

24. Wang, $\mathrm{H}$. et al. New insights into the physical origin of negative capacitance and hysteresis in NCFETs. In2018 IEEE Int. Elec. Dev. Meeting. 31.1.1-31.1.4 (IEEE, San Francisco, 2018).

25. Li, K. et al. Sub-60mV-swing negative-capacitance FinFET without hysteresis In 2015 IEEE Int. Elec. Dev. Meeting. 22.6.1-22.6.4 (IEEE, Washington, DC, 2015).

26. Lee, M. H. et al. Prospects for ferroelectric HfZrOx FETs with experimentally $\mathrm{CET}=0.98 \mathrm{~nm}, \mathrm{SS}_{\text {for }}=42 \mathrm{mV} / \mathrm{dec}, \mathrm{SS}_{\text {rev }}=28 \mathrm{mV} / \mathrm{dec}$, switch-off $<0.2 \mathrm{~V}$, and hysteresis-free strategies. In 2015 IEEE Int. Elec. Dev. Meeting. 22.5.1-22.5.4 (IEEE, Washington, DC, 2015).

27. Kwon, D. et al. Improved subthreshold swing and short channel effect in FDSOI n-channel negative capacitance field effect transistors. IEEE Electron Device Lett. 39, 300-303 (2018)

28. Zhou, J. et al. Ferroelectric HfZrOx Ge and GeSn PMOSFETs with sub-60 $\mathrm{mV} /$ decade subthreshold swing, negligible hysteresis, and improved $\mathrm{I}_{\mathrm{ds}}$. In 2016 IEEE Int. Elec. Dev. Meeting. 12.2.1-12.2.4 (IEEE, San Francisco, 2016).

29. $\mathrm{Si}, \mathrm{M}$. et al. Steep-slope hysteresis-free negative capacitance $\mathrm{MoS}_{2}$ transistors. Nat. Nanotechnol. 13, 24-28 (2018).

30. $\mathrm{Yu}, \mathrm{Z}$. et al. Negative capacitance $2 \mathrm{D} \mathrm{MoS}_{2}$ transistors with sub- $60 \mathrm{mV} / \mathrm{dec}$ subthreshold swing over 6 orders, $250 \mu \mathrm{A} / \mu \mathrm{m}$ current density, and nearlyhysteresis-free. In 2017 IEEE Int. Elec. Dev. Meeting. 23.6.1-23.6.4 (IEEE, San Francisco, 2017).

31. Liu, Y., Huang, Y. \& Duan, X. Van der Waals integration before and beyond two-dimensional materials. Nature 567, 323-333 (2019).

32. Kang, M. J. et al. Alkylated dinaphtho[2,3-b:2',3'-f]Thieno[3,2-b]Thiophenes (Cn-DNTTs): organic semiconductors for high-performance thin-film transistors. Adv. Mater. 23, 1222-1225 (2011).

33. Peng, B., Huang, S., Zhou, Z. \& Chan, P. K. L. Solution-processed monolayer organic crystals for high-performance field-effect transistors and ultrasensitive gas sensors. Adv. Funct. Mater. 27, 1700999 (2017).

34. Giri, G. et al. Tuning charge transport in solution-sheared organic semiconductors using lattice strain. Nature 480, 504-508 (2011).

35. He, D. et al. Ultrahigh mobility and efficient charge injection in monolayer organic thin-film transistors on boron nitride. Sci. Adv. 3, e1701186 (2017).

36. Terry, S. C. et al. Comparison of a BSIM3V3 and EKV MOSFET model for a $0.5 / \mathrm{spl} \mathrm{mu} / \mathrm{m}$ CMOS process and implications for analog circuit design. IEEE Trans. Nucl. Sci. 50, 915-920 (2003).

37. Zhao, Y. et al. Surface potential-based compact model for negative capacitance FETs compatible for logic circuit: with time dependence and multidomain interaction. In 2019 IEEE Int. Elec. Dev. Meeting. 7.5.1-7.5.4 (IEEE, San Francisco, 2019).

38. Li, S. et al. Nanometre-thin indium tin oxide for advanced high-performance electronics. Nat. Mater. 18, 1091-1097 (2019).

39. Lee, S. \& Nathan, A. Subthreshold Schottky-barrier thin-film transistors with ultralow power and high intrinsic gain. Science 354, 302 (2016).

40. Chang, H.-Y. et al. High-performance, highly bendable $\mathrm{MoS}_{2}$ transistors with high-K dielectrics for flexible low-power systems. ACS Nano 7, 5446-5452 (2013).

41. Peng, B. et al. Crystallized monolayer semiconductor for ohmic contact resistance, high intrinsic gain, and high current density. Adv. Mater. 32 2002281 (2020)

42. $\mathrm{Yu}, \mathrm{Z}$. et al. Toward high-mobility and low-power $2 \mathrm{D} \mathrm{MoS}$ field-effect transistors. In 2018 IEEE Int. Elec. Dev. Meeting. 22.4.1-22.4.4 (IEEE, San Francisco, 2018)

43. Kim, D.-H. et al. Epidermal electronics. Science 333, 838 (2011). 
44. Chung, H. U. et al. Binodal, wireless epidermal electronic systems with in-sensor analytics for neonatal intensive care. Science 363, eaau0780 (2019).

45. Ershad, F. et al. Ultra-conformal drawn-on-skin electronics for multifunctional motion artifact-free sensing and point-of-care treatment. Nat. Commun. 11, 3823 (2020).

46. Lee, S. et al. Ultrasoft electronics to monitor dynamically pulsing cardiomyocytes. Nat. Nanotechnol. 14, 156-160 (2019).

47. Sekitani, T. et al. Ultraflexible organic amplifier with biocompatible gel electrodes. Nat. Commun. 7, 11425 (2016)

48. Sugiyama, M. et al. An ultraflexible organic differential amplifier for recording electrocardiograms. Nat. Electron. 2, 351-360 (2019).

49. Fiore, V. et al. An integrated 13.56-MHz RFID Tag in a printed organic complementary TFT technology on flexible substrate. IEEE Trans. Circuits Syst. I: Regul. Pap. 62, 1668-1677 (2015).

\section{Acknowledgements}

This work is supported by National Natural Science Foundation of China 61927808, 61521001, 61734003, 61851401, 91964202, 61861166001, 51861145202, 61821091, 61888102, 61825403 and 11922215; Natural Science Foundation of Jiangsu Province BK20202005; Strategic Priority Research Program of Chinese Academy of Sciences XDB 30000000; General Research Fund (GRF) HKU 17264016, HKU 17204517; Key Laboratory of Advanced Photonic and Electronic Materials, Collaborative Innovation Center of Solid-State Lighting and Energy-Saving Electronics, and the Fundamental Research Funds for the Central Universities, China.

\section{Author contributions}

X.W. conceived and supervised the project. Z.L., B.P., J.Z., Z.Y., D.H., H.N., W.M., W.H., X.C., W.L., D.P., X.T., X.H., Y.S. X.F. and P.K.L.C. contributed to sample preparation, characterization, device fabrication, measurements and data analysis. Y.Z., J.W., L.L. and M.L. carried out circuit simulation and theoretical analysis. Z.L., J.Z., J.X., R.L., Y.Y. and D.S. performed the electrocardiogram test. Z.M. and L.P. fabricated and tested pulse sensors. K.C. and Y.L. performed TEM and data analysis. Z.L., Z.Y. and X.W. co-wrote the manuscript with input from other authors. All authors contributed to discussions.

\section{Competing interests}

The authors declare no competing interests.

\section{Additional information}

Supplementary information The online version contains supplementary material available at https://doi.org/10.1038/s41467-021-22192-2.

Correspondence and requests for materials should be addressed to P.K.L.C. or X.W.

Peer review information Nature Communications thanks Yong-Young Noh and the other, anonymous, reviewer(s) for their contribution to the peer review of this work

Reprints and permission information is available at http://www.nature.com/reprints

Publisher's note Springer Nature remains neutral with regard to jurisdictional claims in published maps and institutional affiliations.

cc (i) Open Access This article is licensed under a Creative Commons Attribution 4.0 International License, which permits use, sharing, adaptation, distribution and reproduction in any medium or format, as long as you give appropriate credit to the original author(s) and the source, provide a link to the Creative Commons license, and indicate if changes were made. The images or other third party material in this article are included in the article's Creative Commons license, unless indicated otherwise in a credit line to the material. If material is not included in the article's Creative Commons license and your intended use is not permitted by statutory regulation or exceeds the permitted use, you will need to obtain permission directly from the copyright holder. To view a copy of this license, visit http://creativecommons.org/ licenses/by/4.0/.

(C) The Author(s) 2021 